\title{
PFA2D Model for Smart Water Management
}

\author{
Roma A. Kudale \\ Asst.Professor \\ Department of Computer Engg, \\ SKN College of Engg \\ Pune,Maharashtra, India
}

\author{
Trupti H. Gurav \\ Asst.Professor \\ Department of Computer Engg, \\ SKN College of Engg \\ Pune, Maharashtra, India
}

\begin{abstract}
Water is essential for life as well as for the economy. Nowadays, to solve and manage the water scarcity is becoming challenging. With the reduction of water resources, the demand for quality water is increasing day by day. Water utilities are important to provide good quality water, but due to outdated or lack of sufficient infrastructure it becomes difficult. Along with this, water get wasted due to metering errors, leaks, pipe bursts, and becomes polluted because of industrial waste. To manage all these issues, water utilities have to come up with smart water management solutions and techniques. This paper proposes a mechanism that predicts the amount of water that can be made available for consumption, distribution, storage of Agriculture, Domestic, Industrial purposes. This mechanism takes into consideration four important factors - current population, current water levels from water reserves, amount of rainfall, amount of water currently consumed. By this mechanism, it will be possible to make predictions as to, how much water should be made available based on the available statistics.
\end{abstract}

\section{Keywords}

IoT, ,smart water management, water problem.

\section{INTRODUCTION}

As we know that water is natural resource and we cannot produce it but we can use it properly. As per survey, Irrigated agriculture and industry use about $70 \%$ and $23 \%$, respectively, of the water used worldwide, while households only use $8 \%$. More region face problem of droughts, drastic seasonal changes, evaporation and rapid population growth. Because of these, growing population face challenge of finite water resources. Also lack of fresh water is becoming hurdle for economic progress and social stability.

We can propose a mechanism that will predicts the amount of water that can be made available for consumption, distribution, storage for different purposes like industrial, domestic and agriculture. This mechanism takes into consideration four important factors - Current population, Current water levels from Water reserves, Amount of Rainfall, Amount of water currently consumed. By this mechanism, it will be possible to make predictions as to, how much water should be made available based on the available statistics. This mechanism is useful in the sense, that it is proposed for all the regions of the entire state, which will enable only authorized access. It will help the concerned officials to take decisions well in advance in order to provide for the future needs. It will also enable the businesses, which use water as a raw material, to predict the amount of such products to be manufactured in order to avoid losses. Using this mechanism, water management can be done effectively.
The major factor in water shortage is its supply and demand. To know how much water level is available and how much we can distribute can be predicted with the tool. One of such analysing tool is SImulation of Water Management in the Arab Republic of Egypt (SIWARE). The SIWARE is a powerful predictive tool for analysing different water policies and management on regional scale, particularly long-term impacts of severe water supply reductions or drought conditions [1].

The paper is organized as follows: section 2 provides the related work section 3 explains proposed system, section 4 explain system architecture with diagram, section 5 advantages, disadvantages and applications and section 6 conclusions.

\section{RELATED WORK}

In paper [1], in many developing countries like India, industrialization, urbanization and population growth has led to increased water consumption and pollution. According to the World Bank report released in 2014, urban water supply in India is faced with severe challenges including distribution inefficiency leading to higher operational costs with only $20 \%$ of the connections being metered, and in most cities about $40 \%$ water supply not resulting in any revenue. Hence, the traditional water metering system employed in India needs both infrastructural improvements and a smart flow metering approach. The manual examination of water meters for billing purposes is prone to human error and manipulation.

In [2], Ningbo is a city famous for rich water resources, drinking water sources are regarded as the most important strategic resources which is closely related to the quality of people's life of the whole city as well as the stability of economy. Water quality of these reservoirs is undoubtedly affected by the production and everyday life in the surrounding towns and villages which hold a population varying from hundreds to thousands. In order to ensure the security of the drinking water and standardize the production, it's necessary to delineate the conservation regions. In this paper, the 3D simulation technology combined with various data is successfully applied to the delineation of drinking water source protection areas of reservoirs. On this basis, the 3D display system of Ningbo drinking water source protection areas is built to effectively promote safety supervision of these zones and ensure the security of the drinking water of the inhabitants.

In this paper[3], they have described two types of collaboration platforms: one for SCWA in California and the other for the Marine Institute of Ireland. Both instances differ in the types and use of data collected and presented. They also differ in the applications they serve water supply and management in the case of SCWA versus marine and coastal environmental monitoring, protection, and management for SmartBay Galway. Despite these differences, the projects 
highlight and demonstrate that collecting, sharing, visualizing, and analyzing data in a collaborative format can enhance the opportunity for better decision making. In addition, these smarter water management techniques provide a vehicle to uncover new opportunities for innovation using information technology, opportunities that might have been otherwise missed.

In [4], There are already numbers of approaches to predicting the water quantity in the future, such as $\operatorname{GM}(1,1)$ model, Regression Analysis, Time Series Analysis, Artificial Neural Networks (ANN), MGM $(1, n)$, etc. XU and YUAN et al.apply the grey system theory to forecast the annual water demand with GM $(1,1)$ model, during which the characters of yearly demand data series of water-distribution systems are analysed. However, prediction models based on

GM $(1,1)$ are univariate and can be difficult to reflect the complicated relationships between annual water consumption and its main impact factors. HUANG applies the coupling model of Grey-system and multiple stepwise regressions to predict the water-consumed quantity, with the result showing the main factors for the change of total water quantity. Also, a self-adapting MGM $(1, n)$ model is presented to take multi variables into account in water consumption by Lou et al. Zhang et al provide a time sequence model of water resources in Gansu Province and analyse the change pattern describing the periodic change trend.

This paper [5], has described an on-going implementation of a GIS-based DSS for water management stake-holders at the river basin level ("level 2" in the INGHA terminology). The software is taking advantage of the latest technologies available on the market (ArcGIS 10 Server with specialized modeling packages - ArcHydro [1], SQL Server 2012) and most advanced SCADA systems available at ANAR. This tool will be useful in both extreme conditions - floods and draughts.

\section{PROPOSED SYSTEM}

The Water cycle (water resource, production, distribution, consumption, collection and treatment of waste water) play an integral part of the urban system, influencing each pillar of the urban society and its functionality, sustaining populations, generating energy, supporting tourism and recreational activities, ensuring environmental and human health, and fueling local economic development. Such increasing convergence fosters urban growth, as more than half of the world's population currently reside in urban areas.

Our Approach suggests that achieving water security objectives means maintaining acceptable levels for four water risks: risk of shortage (including droughts), risk of inadequate quality, risk of excess (including floods), and risk of undermining the resilience of freshwater systems (e.g. by exceeding the coping capacity of the surface and groundwater bodies). This approach evidences an increasing awareness of the importance of tackling water-related challenges from an integrated, holistic perspective, considering both acceptable levels of risks, as well as their potential consequences (economic, environmental, social) on urban stakeholders. Flow of the system can be given as shown in Fig 1. Flow consists of majorly 6 steps. Those are 1) Data Pre-processing 2) Data Filtering 3) Data Aggregation 4) Data Analysis 5) Decision Model 6) Result means prediction of water management. Thus we refer our proposed model as PFA2D Model. This result can be used for different purposes which are explained at Application section.

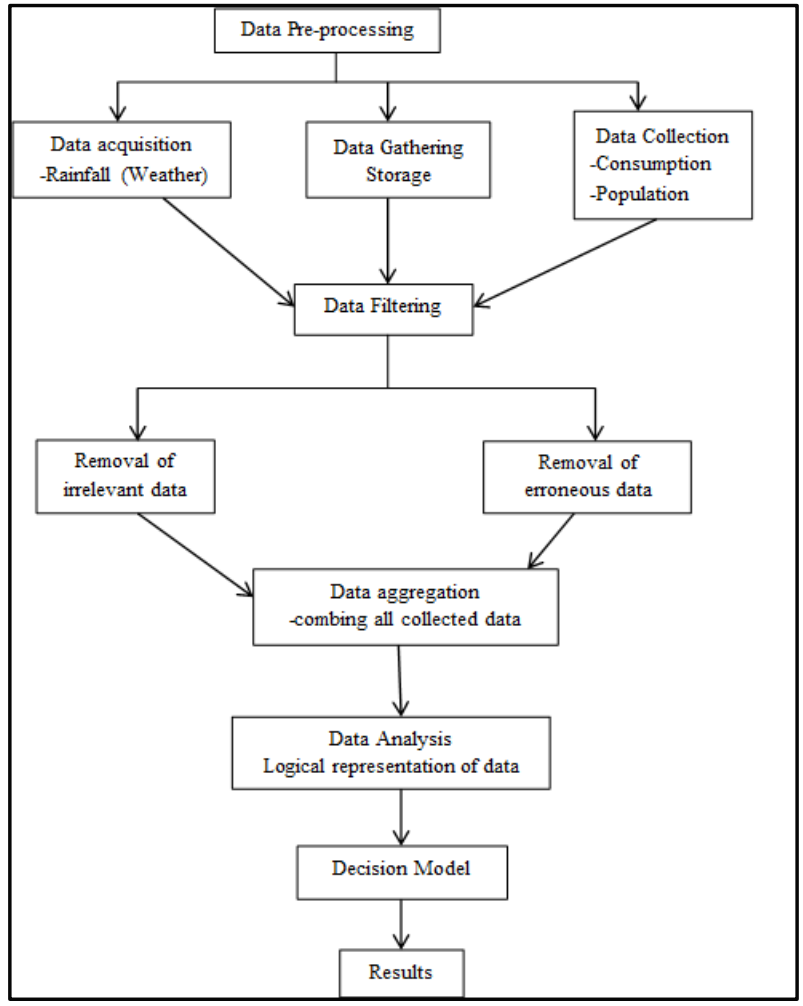

Fig 1: Flow of Model

\section{SYSTEM ARCHITECTURE}

System architecture is of three tire architecture. It includes three major steps like Water Management Data Acquisition Unit (WMDAU), Data Processing Unit (DPU), Data Analysis and Decision Unit (DADU). It is shown in Fig 2.

\subsection{A Water Management Data Acquisition Unit (WMDAU)}

Here system needs to consider data of population, consumption, storage and rainfall as big data. Obtaining the data from database related to population, consumption, storage and rainfall promotes the expansion of water management system. For efficient predictions, there is the necessity of parallel processing of the data related to population, consumption, storage and rainfall .The parallel processing of all four factors saves the time and helps to take instant decision related to water distribution. System needs data related to above four factors year wise, as it will take decision for prediction. It will depend on the weekly data so, consider week as a unit. For that first find out ratio which gives information of how much data is required. To find out ratio use parallel processing technique. By analysing the big data related to population, consumption, storage and rainfall in parallel we can find out efficient ratio.

The data obtained is unstructured or structured data. System need to convert structured data to unstructured data so that we can handle it in parallel using NoSQL database techniques.

Some relational data pre-processing techniques are data integration, data cleaning, and redundancy elimination. After pre-processing phase, the collected data are provided to the data Analysis and decision making system.

\subsection{Data Processing Unit}

In data processing unit (DPU), the filtration and load balancer units have two basic responsibilities, such as filtration of data 
and load balancing of processing power. Filtration identifies the useful data for analysis since it only allows useful information, whereas the rest of the data are blocked and are discarded. Hence, it results in enhancing the performance of the whole proposed system. Apparently, the load-balancing part of the server provides the facility of dividing the whole filtered data into parts and assign them to various processing servers. The filtration and load-balancing algorithm varies from analysis to analysis; e.g., if there is only a need for analysis of rainfall and population data, the measurement of these described data is filtered out, and is segmented into parts.

Each processing server has its algorithm implementation for processing incoming segment of data from Filtration and Load Balancing System (FLBS). Each processing server makes statistical calculations, any measurements, and performs other mathematical or logical tasks to generate intermediate results against each segment of data. Since these servers perform tasks independently and in parallel, the performance of proposed system is dramatically enhanced, and the results against each segment are generated in real time. The results generated by each server are then sent to the aggregation server for compilation, organization, and storing for further processing

\subsection{Data Analysis and Design Unit}

DADU contains three major portions, such as aggregation and compilation unit, results storage unit(s), and decision making unit. When the results are ready for compilation, the processing units in DPU send the partial results to the aggregation and compilation unit, since the aggregated results are not in organized and compiled form. Therefore, there is a need to aggregate the related results and organized them into a proper form for further processing and to store them. In the proposed architecture, aggregation and compilation unit is supported by various algorithms that compile, organize, store, and transmit the results. Again, the algorithm varies from requirement to requirement and depends on the analysis needs.

Aggregation unit stores the compiled and organized results into the result's storage with the intention that any unit can use it as it can process at any time. The aggregation unit also sends the same copy of that result to the decision-making unit to process that result for making decision. The decisionmaking unit is supported by the decision algorithm, which inquires different things from the result, and then makes various decisions (e.g., in our analysis, we analyse population, consumption, storage and rainfall, whereas other finding such as humidity, wastage, distribution uses can also be found). The decision algorithm must be strong and correct enough that efficiently produce results to discover hidden things and make decisions. The decision part of the architecture is significant since any small error in decision-making can degrade the efficiency of the whole analysis. DADU finally displays or broadcasts the decisions, so that any application can utilize those decisions at real time to make their development. The applications can be any business software, general purpose community software, or other social networks that need those findings (i.e., decision-making).

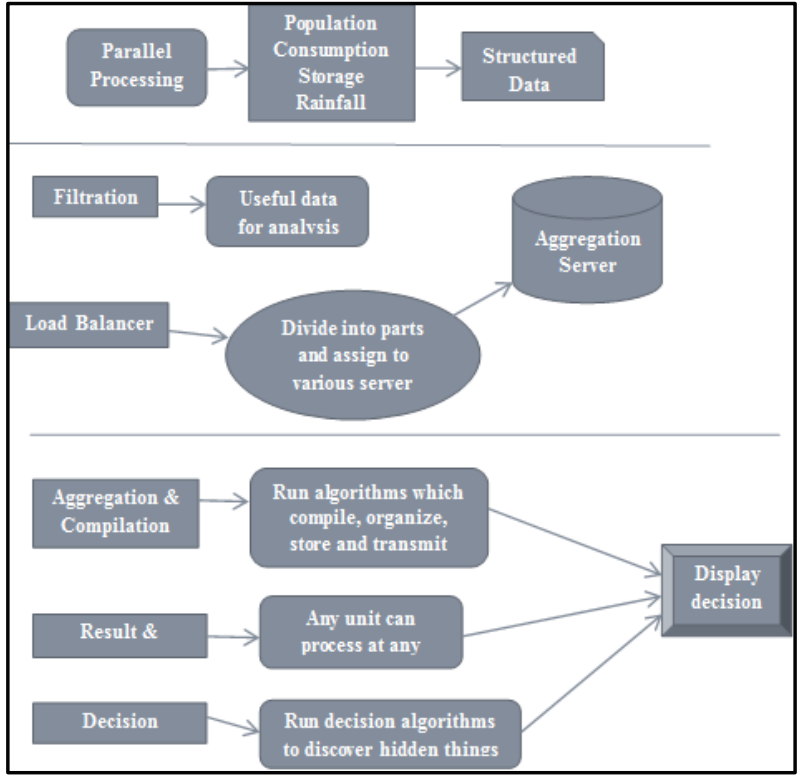

Fig 2: System Architecture

\section{ADVANTAGES/ DISADVANTAGES}

There are different advantages, disadvantages and applications. Those are explained in next part.

\subsection{Advantages}

1. Gives complete details of the water table of each region.

2. Help the administrative officer to take decisions related to water management.

3. This enables judicious supply of water in each region.

4. Instant decision making is possible for supplying water.

5. Even though only higher Govt. authorities can take decision based on this mechanism, the common people can also visit this page.

\subsection{Disadvantages}

1. In some uncertain condition, it is difficult to take decision as only four factors viz population, consumption, storage and rainfall, are deal with.

2. There is a possibility of unethical, irrelevant and noisy data while collecting inputs from the Internet.

\subsection{Applications}

1. Used by the authorities of the particular region to make decisions regarding storage, distribution and consumption.

2. For industries, it helps to make decisions about product manufacturing.

3. Helps to take decisions in the field of agriculture, that is by looking at the heat map, decisions about which crop is to be planted depending upon its water requirements and the available water.

\section{CONCLUSION}

Water Scarcity is one of the major concerns before us today. There is a huge gap between Demand \& Supply of water which results in unequal distribution of water in different 
areas. This is because there is a lack of a mechanism that will predict consumption of water and promote the government to take decisions for judicious supply of water. So in this paper we propose such mechanism which will predict how much water level is left. Accordingly we can take decision according to the application. This paper highlights only four important factors - current population, current water levels from water reserves, amount of rainfall, amount of water currently consumed. In future we can expand it by considering more factors.

\section{REFERENCES}

[1] Wael M. Khairy, M. Safwat Abdel-Dayem, Tommy L. Coleman," DETERMINING A BETTER WATER MANAGEMENT USING A GEOGRAPHICAL TECHNIQUE - A CASE STUDY IN EGYPT" 2001 IEEE.

[2] Anjana S, Sahana M N, Ankith S, K Natarajan4, K R Shobha " An IoT based 6LoWPAN enabled Experiment for Water Management" at IEEE ANTS 2015.

[3] Weimin Chen, Jia Liao , Xiuyun Wu, Kun Wang," Research and Application of 3D GIS Technology on the Delineation and Management of Urban Drinking Water Source Protection Area" at Intrenational Symposium on Geomatics for integrated water resource management (GIWRM) 2012.

[4] C. E. Hidaka J. Jasperse H. R. Kolar R. P. Williams, "Collaboration platforms in smarter water management" at IBM journal march 2011
[5] Cuiyun Gao, Linbo Jin, Wanggen Wan, Rui Wang," GANN-BASED PREDICTION OF FRESH WATER RESOURCES" at IET International conference on smart and sustainable city 2013 , pages $79-83$

[6] Marius RATA, Tudor Viorel BLIDARU, Dr. Paul Serban AGACHI," GIS Data Modeling -in Water Management Decision Support Systems" at IEEE conference 2014 .

[7] Zifu Li*, W. F. Geiger**, Xin Jin*,'New Concept for Water Management in Urban Areas of China" at IEEE, 2009.

[8] Simei Zhang," The overview of prediction methods of urban domestic water consumption", research article at Journal of Chemical and Pharmaceutical Research, 2013, 5(9):416-419.

[9] B Dr. Prakash Somalkar," WATER MANAGEMENT IN MAHARASHTRA" at Abhinav National monthly refereed journal of research in commerce and management, Volume no.1, Issue no.11, ISSN 2277 1166.

[10] Haiquan Yangl,2,Dingtao Shenl,2,Huang Huang," A WebGIS-based Monitoring and Early-warning System for Geological Disasters" at $23^{\text {rd }}$ international conference on geo-informatics 2015. 